\title{
$k$-Steps Explicit Iterative Algorithms to Solve Generalized System of Nonlinear Mixed Variational Inequalities
}

\author{
Sanjeev Gupta* \\ Department of Mathematics \\ GLA University \\ Mathura-281406, India \\ Email address: guptasanmp@gmail.com
}

Received: 01 June, 2020 / Accepted: 20 January, 2021 / Published online: 22 February, 2021

\begin{abstract}
The objective of this paper is to consider new generalized systems of nonlinear mixed variational inequalities including $3 k$-distinct nonlinear relaxed cocoercive operators. We proposed $k$-steps explicit iterative methods including projection operators for this considered system and present the equivalent fixed point problem of this new generalized systems of variational inequalities. This equivalent fixed point problem suggest to us $k$-steps explicit iterative algorithms to obtain an approximate solution of the considered system. Convergence results of $k$-step explicit iterative algorithms are obtained.
\end{abstract}

\begin{abstract}
AMS (MOS) Subject Classification Codes: 49J40; 90C33
Key Words: $k$-steps explicit iterative algorithm, Generalized system of nonlinear mixed variational inequalities, Projection, Relaxed $(\alpha, \beta)$-cocoercitivity, $\kappa$-Lipschitz continuity.
\end{abstract}

\section{Introduction and Preliminaries}

The field of variational inequalities has appealed a lot of consideration. Since a number of real-world problems in optimal control, decision theory, game theory, physics, economics, management sciences, engineering problems can be formulated as nonlinear variational inequalities, see for example [1]. In this paper, we consider and study the generalized system of nonlinear mixed variational inequalities.

There are several techniques to solved variation inequality problems. The projection technique is one of the iterative methods to solve variational inequalities. Many heuristics widely used projection techniques to solve variational inequalities and systems of variational inequalities. Recently Noor et. al [8, 13], Verma [15, 16, 17], Hao et. al [5], Kim [6] and Zhang [19] obtained the approximate solution of the system of nonlinear variational 
inequalities by using two or three steps iterative methods involving projection operator. For further details, please see $[2,3,4,5,6,8,12,13,15,16,17,19]$.

This present work is impelled by the research going on this field. The aim is to perusal the new generalized system of nonlinear variational inequalities connecting with $3 k$-distinct nonlinear relaxed $(r, s)$-cocoercive operators. First, we give the fixed point problem equivalent to the variational considered system. By this equivalent formulations, we propose $k$-steps explicit algorithms with projection operators. Utilization of projection approach, we attempt to obtain an approximate solution of the generalized system of nonlinear variational inequalities. The consider conditions guaranteed the convergence of iterative sequences dstobtained by the $k$-steps explicit algorithms. This work extends and improves the well-known results in the literature $[5,8,9,15,16,17,19]$.

Throughout the manuscript, $\mathcal{H}$ is a real Hilbert space endowed with a norm $\|$.$\| and an$ inner product $\langle.,$.$\rangle . Let \mathcal{D} \subset \mathcal{H}$ be a closed and convex set in $\mathcal{H}$.

Let us given that nonlinear operators $\mathcal{A}: \mathcal{D} \rightarrow \mathcal{H}, f, g: \mathcal{D} \rightarrow \mathcal{D}$, then generalized mixed variational inequality problem (GMVIP) is to find $p^{*} \in \mathcal{D}$ such that

$$
\left\langle\lambda \mathcal{A}\left(p^{*}\right)+g\left(p^{*}\right)-f\left(p^{*}\right), p-g\left(p^{*}\right)\right\rangle \geq 0, \forall p \in \mathcal{D}, \lambda>0 .
$$

Lemma 1.1 [7] For given $q^{*} \in \mathcal{H}, p^{*} \in \mathcal{D}$ satisfies the inequality

$$
\left\langle p^{*}-q^{*}, p-p^{*}\right\rangle \geq 0, p \in \mathcal{D}
$$

if and only if $p^{*}=\mathcal{P}_{\mathcal{D}}\left(q^{*}\right)$ where $\mathcal{P}_{\mathcal{D}}$ is the projection from $\mathcal{H}$ onto $\mathcal{D}$.

By Lemma (1.1), it can be showed that $p^{*}$ is a solution of GMVIP (1.1) if and only if $p^{*}$ is a fixed point of $I-g+\mathcal{P}_{\mathcal{D}}[f-\lambda \mathcal{A}]$, where $I$ is identity mapping.

If $f=g$, then the GMVIP (1.1) is equivalent to find $p^{*} \in \mathcal{D}$ such that

$$
\left\langle\mathcal{A}\left(p^{*}\right), p-g\left(p^{*}\right)\right\rangle \geq 0, \forall p \in \mathcal{D} .
$$

If $f=g=I$, then the GMVIP (1.1) is equivalent to find $p^{*} \in \mathcal{D}$ such that

$$
\left\langle\mathcal{A}\left(p^{*}\right), p-p^{*}\right\rangle \geq 0, \forall p \in \mathcal{D},
$$

known as classical variational inequality, proposed by Stampacchia [14].

If $\mathcal{D}^{*}=\{p \in \mathcal{H}:\langle p, q\rangle \geq 0, \forall q \in \mathcal{D}\}$ is a polar cone of convex cone $\mathcal{D}$ in $\mathcal{H}$. Then Problem (1.2) is equivalent to general nonlinear complementarity problem to find $p^{*} \in D$ such that

$$
\mathcal{A}\left(p^{*}\right) \in \mathcal{D}^{*} \text { and }\left\langle\mathcal{A}\left(p^{*}\right), g\left(p^{*}\right)\right\rangle=0 .
$$

Definition 1.2 [16] A mapping $\mathcal{A}: \mathcal{D} \rightarrow \mathcal{H}$

(i) is monotone if

$$
\left\langle\mathcal{A}\left(p^{1}\right)-\mathcal{A}\left(p^{1 *}\right), p^{1}-p^{1 *}\right\rangle \geq 0, \forall p^{1}, p^{1 *} \in \mathcal{D} ;
$$


(ii) is $\mu$-strongly monotone if $\exists$ a constant $\mu>0$ such that

$$
\left\langle\mathcal{A}\left(p^{1}\right)-\mathcal{A}\left(p^{1 *}\right), p^{1}-p^{1 *}\right\rangle \geq \mu\left\|p^{1}-p^{1 *}\right\|^{2}, \forall p^{1}, p^{1 *} \in \mathcal{D} ;
$$

(iii) is relaxed $\bar{r}$-cocoercive if $\exists$ a constant $\bar{r}>0$ such that

$$
\left\langle\mathcal{A}\left(p^{1}\right)-\mathcal{A}\left(p^{1 *}\right), p^{1}-p^{1 *}\right\rangle \geq-\bar{r}\left\|\mathcal{A}\left(p^{1}\right)-\mathcal{A}\left(p^{1 *}\right)\right\|^{2}, \forall p^{1}, p^{1 *} \in \mathcal{D} ;
$$

(iv) is relaxed $(\bar{r}, \bar{s})$-cocoercive if $\exists$ constants $\bar{r}, \bar{s}>0$ such that

$$
\left\langle\mathcal{A}\left(p^{1}\right)-\mathcal{A}\left(p^{1 *}\right), p^{1}-p^{1 *}\right\rangle \geq-\bar{r}\left\|\mathcal{A}\left(p^{1}\right)-\mathcal{A}\left(p^{1 *}\right)\right\|^{2}+\bar{s}\left\|p^{1}-p^{1 *}\right\|^{2}, \forall p^{1}, p^{1 *} \in \mathcal{D} ;
$$

(v) is $\bar{t}$-Lipschitz continuous if $\exists$ a constant $\bar{t}>0$ such that

$$
\left\|\mathcal{A}\left(p^{1}\right)-\mathcal{A}\left(p^{1 *}\right)\right\| \leq \bar{t}\left\|p^{1}-p^{1 *}\right\|, \forall p^{1}, p^{1 *} \in \mathcal{D} .
$$

(vi) is nonexpansive

$$
\left\|\mathcal{A}\left(p^{1}\right)-\mathcal{A}\left(p^{1 *}\right)\right\| \leq\left\|p^{1}-p^{1 *}\right\|, \forall p^{1}, p^{1 *} \in \mathcal{D} .
$$

Here, $\mathcal{K}(\mathcal{A})$ represent the set of all fixed point of mapping $\mathcal{A}$.

Definition $1.3[10,11]$ Let $\mathcal{D} \subset \mathcal{H}$ be a convex subset in $\mathcal{H}$ and $\mathcal{A}: \mathcal{D} \rightarrow \mathcal{H}$, then (i): $\mathcal{D}$ is l-convex if $\exists$ a function $l: \mathcal{D} \rightarrow \mathcal{D}$ such that

$$
l(p)+\lambda(q-l(p)) \in \mathcal{D}, \forall p, q \in \mathcal{D}, \text { where } \lambda \in(0,1) ;
$$

(ii) $\mathcal{A}$ is l-convex if $\exists$ a function $l: \mathcal{D} \rightarrow \mathcal{D}$ such that

$$
\mathcal{A}(l(p)+\lambda(q-l(p))) \leq(1-\lambda) \mathcal{A}(l(p))+\lambda \mathcal{A}(q), \forall p, q \in \mathcal{D}, \text { where } \lambda \in(0,1) .
$$

Let $\mathcal{A}_{i}: \underbrace{\mathcal{D} \times \mathcal{D} \times \ldots \times \mathcal{D}}_{(\mathrm{k} \text { times })} \rightarrow \mathcal{H}$ and $f_{i}, g_{i}: \mathcal{D} \rightarrow \mathcal{D}$ be $3 k$-distinct nonlinear operators for each $i \in\{1,2, \ldots, k\}$. Then generalized system of nonlinear mixed variational inequalities problem (GSNMVIP) is to find $\left(p^{1}, p^{2}, \ldots p^{k}\right) \in \underbrace{\mathcal{D} \times \mathcal{D} \times \ldots \times \mathcal{D}}_{(\mathrm{k} \text { times })}$ such that

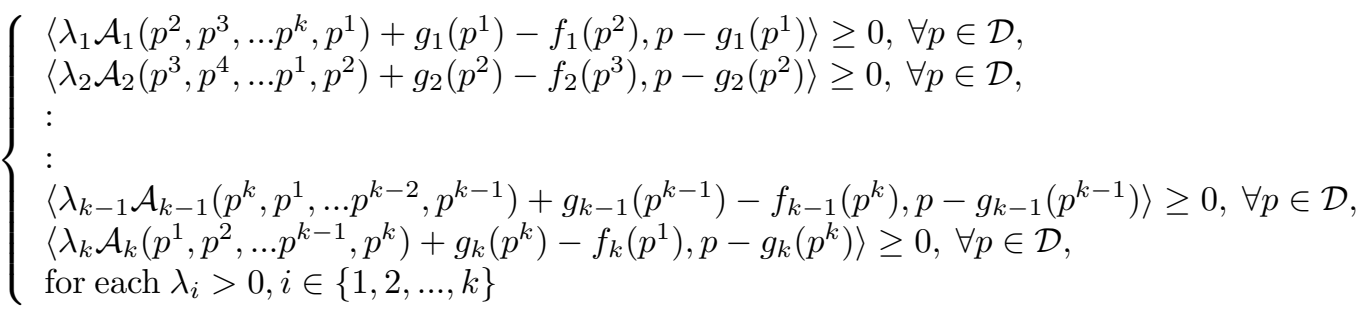


Let $\mathcal{D}_{1}(\neq \emptyset)$ is the solution set of GSNMVIP (1.5). Lemma (1.1) permits us to write GSNMVIP (1.5) equivalently to fixed point problem as follows:

$$
\left\{\begin{array}{l}
g_{1}\left(p^{1}\right)=\mathcal{P}_{\mathcal{D}}\left[f_{1}\left(p^{2}\right)-\lambda_{1} \mathcal{A}_{1}\left(p^{2}, p^{3}, \ldots p^{k}, p^{1}\right)\right] \\
g_{2}\left(p^{2}\right)=\mathcal{P}_{\mathcal{D}}\left[f_{2}\left(p^{3}\right)-\lambda_{2} \mathcal{A}_{2}\left(p^{3}, p^{4}, \ldots p^{1}, p^{2}\right)\right] \\
: \\
: \\
g_{k-1}\left(p^{k-1}\right)=\mathcal{P}_{\mathcal{D}}\left[f_{k-1}\left(p^{k}\right)-\lambda_{k-1} \mathcal{A}_{k-1}\left(p^{k}, p^{1}, \ldots p^{k-2}, p^{k-1}\right)\right], \\
g_{k}\left(p^{k}\right)=\mathcal{P}_{\mathcal{D}}\left[f_{k}\left(p^{1}\right)-\lambda_{k} \mathcal{A}_{k}\left(p^{1}, p^{2}, \ldots p^{k-1}, p^{k}\right)\right] \\
\text { for each } \lambda_{i}>0, i \in\{1,2, \ldots, k\} .
\end{array}\right.
$$

The parameters $\lambda_{i}, i \in\{1,2, \ldots, k\}$ have very important role in the convergence analysis of $k$-steps explicit iterative methods. Here, we given some special cases of GSNMVIP (1.5).

(I) If $f_{i}=I$ for each $i \in\{1,2, \ldots, k\}$ then GSNMVIP (1.5) reduces to finding $\left(p^{1}, p^{2}, \ldots p^{k}\right) \in$ $\underbrace{\mathcal{D} \times \mathcal{D} \times \ldots \times \mathcal{D}}_{(\mathrm{k} \text { times })}$ such that

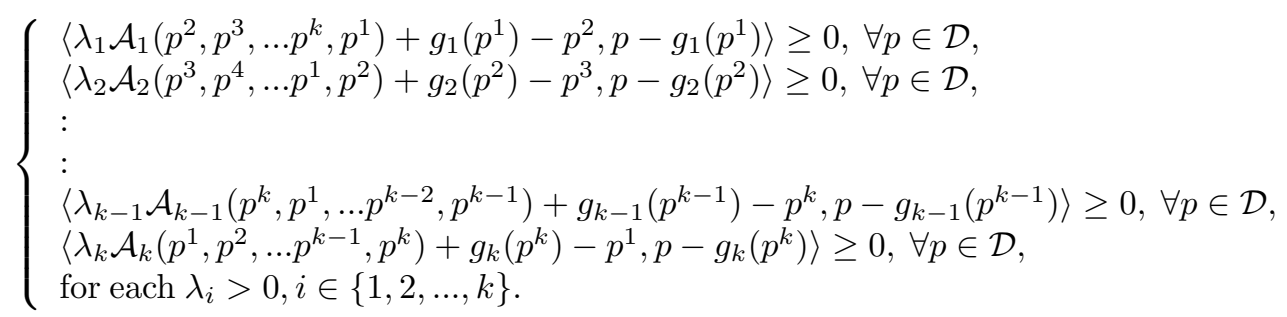

Let $\mathcal{D}_{2}(\neq \emptyset)$ is the solution set of GSNMVIP (1.7). Problem (1.7) is equivalent to fixed point problem as follows:

$$
\left\{\begin{array}{l}
g_{1}\left(p^{1}\right)=\mathcal{P}_{\mathcal{D}}\left[p^{2}-\lambda_{1} \mathcal{A}_{1}\left(p^{2}, p^{3}, \ldots p^{k}, p^{1}\right)\right] \\
g_{2}\left(p^{2}\right)=\mathcal{P}_{\mathcal{D}}\left[p^{3}-\lambda_{2} \mathcal{A}_{2}\left(p^{3}, p^{4}, \ldots p^{1}, p^{2}\right)\right] \\
: \\
: \\
g_{k-1}\left(p^{k-1}\right)=\mathcal{P}_{\mathcal{D}}\left[p^{k}-\lambda_{k-1} \mathcal{A}_{k-1}\left(p^{k}, p^{1}, \ldots p^{k-2}, p^{k-1}\right)\right] \\
g_{k}\left(p^{k}\right)=\mathcal{P}_{\mathcal{D}}\left[p^{1}-\lambda_{k} \mathcal{A}_{k}\left(p^{1}, p^{2}, \ldots p^{k-1}, p^{k}\right)\right] \\
\text { for each } \lambda_{i}>0, i \in\{1,2, \ldots, k\} .
\end{array}\right.
$$

(II) If $g_{i}=I$ for each $i \in\{1,2, \ldots, k\}$ then GSNMVIP (1.5) reduces to finding $\left(p^{1}, p^{2}, \ldots p^{k}\right) \in$ $\underbrace{\mathcal{D} \times \mathcal{D} \times \ldots \times \mathcal{D}}_{(\mathrm{k} \text { times })}$ such that

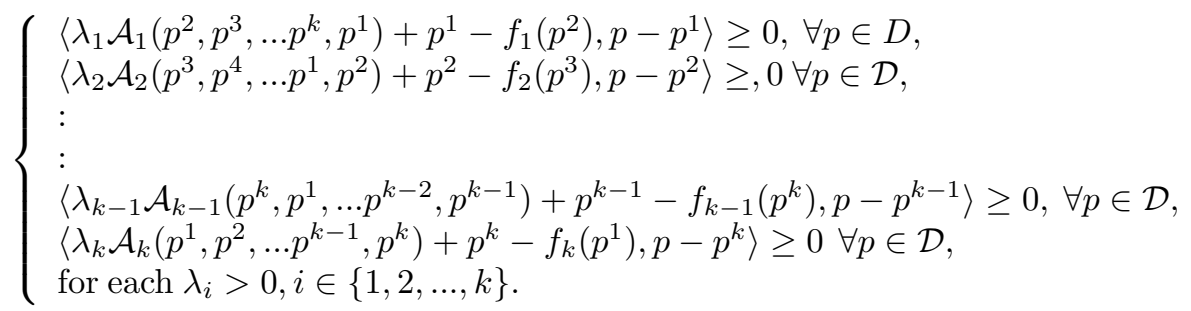


Let $\mathcal{D}_{3}(\neq \emptyset)$ is the solution set of GSNMVIP (1.9). Problem (1.9) is equivalent to fixed point problem as follows:

$$
\left\{\begin{array}{l}
p^{1}=\mathcal{P}_{\mathcal{D}}\left[f_{1}\left(p^{2}\right)-\lambda_{1} \mathcal{A}_{1}\left(p^{2}, p^{3}, \ldots p^{k}, p^{1}\right)\right] \\
p^{2}=\mathcal{P}_{\mathcal{D}}\left[f_{2}\left(p^{3}\right)-\lambda_{2} \mathcal{A}_{2}\left(p^{3}, p^{4}, \ldots p^{1}, p^{2}\right)\right] \\
: \\
: \\
p^{k-1}=\mathcal{P}_{\mathcal{D}}\left[f_{k-1}\left(p^{k}\right)-\lambda_{k-1} \mathcal{A}_{k-1}\left(p^{k}, p^{1}, \ldots p^{k-2}, p^{k-1}\right)\right] \\
p^{k}=\mathcal{P}_{\mathcal{D}}\left[f_{k}\left(p^{1}\right)-\lambda_{k} \mathcal{A}_{k}\left(p^{1}, p^{2}, \ldots p^{k-1}, p^{k}\right)\right] \\
\text { for each } \lambda_{i}>0, i \in\{1,2, \ldots, k\} .
\end{array}\right.
$$

(III) $f_{i}=g_{i}=I$ for each $i \in\{1,2, \ldots, k\}$ then GSNMVIP (1.5) reduces to finding $\left(p^{1}, p^{2}, \ldots p^{k}\right) \in \underbrace{\mathcal{D} \times \mathcal{D} \times \ldots \times \mathcal{D}}_{(\mathrm{k} \text { times })}$ such that

$$
\left\{\begin{array}{l}
\left\langle\lambda_{1} \mathcal{A}_{1}\left(p^{2}, p^{3}, \ldots p^{k}, p^{1}\right)+p^{1}-p^{2}, p-p^{1}\right\rangle \geq 0, \forall p \in \mathcal{D}, \\
\left\langle\lambda_{2} \mathcal{A}_{2}\left(p^{3}, p^{4}, \ldots p^{1}, p^{2}\right)+p^{2}-p^{3}, p-p^{2}\right\rangle \geq 0, \forall p \in \mathcal{D}, \\
: \\
: \\
\left\langle\lambda_{k-1} \mathcal{A}_{k-1}\left(p^{k}, p^{1}, \ldots p^{k-2}, p^{k-1}\right)+p^{k-1}-p^{k}, p-p^{k-1}\right\rangle \geq 0, \forall p \in \mathcal{D}, \\
\left\langle\lambda_{k} \mathcal{A}_{k}\left(p^{1}, p^{2}, \ldots p^{k-1}, p^{k}\right)+p^{k}-p^{1}, p-p^{k}\right\rangle \geq 0, \forall p \in \mathcal{D}, \\
\text { for each } \lambda_{i}>0, i \in\{1,2, \ldots, k\} .
\end{array}\right.
$$

Let $\mathcal{D}_{4}(\neq \emptyset)$ is the solution set of GSNMVIP (1.11). Problem (1.11) is equivalent to fixed point problem as follows:

$$
\left\{\begin{array}{l}
p^{1}=\mathcal{P}_{\mathcal{D}}\left[p^{2}-\lambda_{1} \mathcal{A}_{1}\left(p^{2}, p^{3}, \ldots p^{k}, p^{1}\right)\right] \\
p^{2}=\mathcal{P}_{\mathcal{D}}\left[p^{3}-\lambda_{2} \mathcal{A}_{2}\left(p^{3}, p^{4}, \ldots p^{1}, p^{2}\right)\right] \\
: \\
: \\
p^{k-1}=\mathcal{P}_{\mathcal{D}}\left[p^{k}-\lambda_{k-1} \mathcal{A}_{k-1}\left(p^{k}, p^{1}, \ldots p^{k-2}, p^{k-1}\right)\right] \\
p^{k}=\mathcal{P}_{\mathcal{D}}\left[p^{1}-\lambda_{k} \mathcal{A}_{k}\left(p^{1}, p^{2}, \ldots p^{k-1}, p^{k}\right)\right] \\
\text { for each } \lambda_{i}>0, i \in\{1,2, \ldots, k\}
\end{array}\right.
$$

(IV) If $\mathcal{A}_{i}$, for each $\mathrm{i} \in\{1,2, \ldots, \mathrm{k}\}$ is univariate mappings, then GSNMVIP (1.5) reduces to finding $\left(p^{1}, p^{2}, \ldots p^{k}\right) \in \underbrace{\mathcal{D} \times \mathcal{D} \times \ldots \times \mathcal{D}}_{(\mathrm{k} \text { times })}$ such that

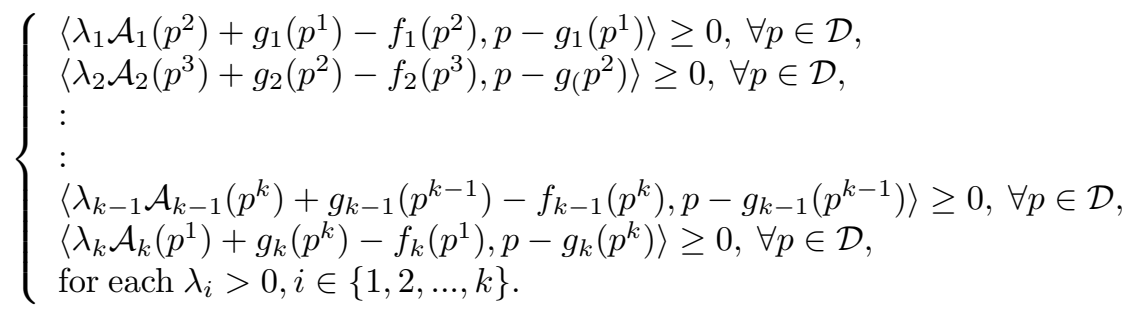


Let $\mathcal{D}_{5}(\neq \emptyset)$ is the solution set of GSNMVIP (1.13). Problem (1.13) is equivalent to fixed point problem as follows:

$$
\left\{\begin{array}{l}
g_{1}\left(p^{1}\right)=\mathcal{P}_{\mathcal{D}}\left[f_{1}\left(p^{2}\right)-\lambda_{1} \mathcal{A}_{1}\left(p^{2}\right)\right] \\
g_{2}\left(p^{2}\right)=\mathcal{P}_{\mathcal{D}}\left[f_{2}\left(p^{3}\right)-\lambda_{2} \mathcal{A}_{2}\left(p^{3}\right)\right] \\
: \\
: \\
g_{k-1}\left(p^{k-1}\right)=\mathcal{P}_{\mathcal{D}}\left[f_{k-1}\left(p^{k}\right)-\lambda_{k-1} \mathcal{A}_{k-1}\left(p^{k}\right)\right] \\
g_{k}\left(p^{k}\right)=\mathcal{P}_{\mathcal{D}}\left[f_{p}\left(p^{1}\right)-\lambda_{k} \mathcal{A}_{k}\left(p^{1}\right)\right] \\
\text { for each } \lambda_{i}>0, i \in\{1,2, \ldots, k\} .
\end{array}\right.
$$

(V): If $\mathcal{D}$ is closed convex cone of $\mathcal{H}$, then GSNMVIP (1.5) reduces to complementarity problem (1.14) to finding $\left(p^{1}, p^{2}, \ldots p^{k}\right) \in \underbrace{\mathcal{D} \times \mathcal{D} \times \ldots \times \mathcal{D}}_{(\mathrm{k} \text { times })}$ such that

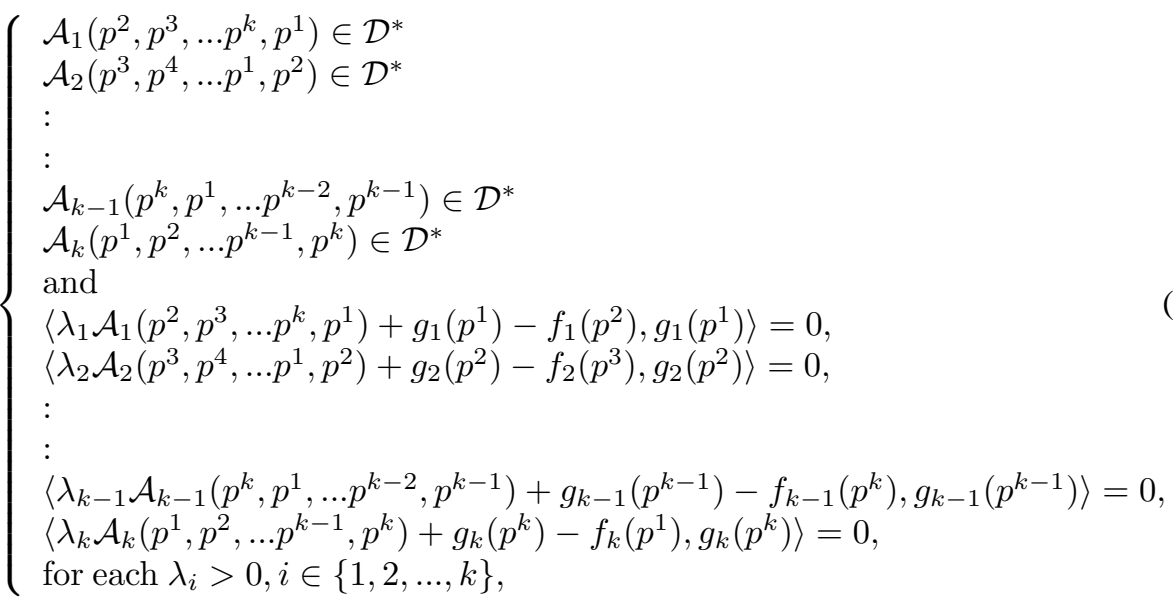

where $D^{*}=\{g \in \mathcal{H}:\langle g, q\rangle \geq 0, \forall q \in \mathcal{D}\}$ is polar cone to $\mathcal{D}$. Let $\mathcal{D}_{6}$ is the solution set of GSNMVIP (1.15).

Remark 1.4 If $k=1$ and $g_{i}=I$, then problem (1.5) reduces to variational inequalities problem discussed by Noor in [9]. If $k=1,2, A_{i}=A$ and $f_{i}=g_{i}=I$, then problem (1.5) reduces to system of variational inequalities problem discussed by Verma in [16]. In addition, $A_{i}$ is univariate mapping then problem (1.5) reduces to system of variational inequalities problem discussed by Verma in $[15,17]$. If $k=1,2$, and $f_{i}=I$, then problem (1.5) reduces to system of variational inequalities problem discussed by Noor in [8]. If $k=1,2$, and $f_{i}=g_{i}$, then problem (1.5) reduces to system of variational inequalities problem discussed by Hao in [5]. If $k=1,2,3$, then problem (1.5) reduces to system of variational inequalities problem discussed by Zhangs in [19].

\section{Explicit k-Steps Iterative Algorithms}

Here, we put forward the k-steps explicit iterative algorithms to find the approximate solution of GSNMVIP (1.5) by using its alternative equivalent fixed point formulations (1.6). 
Let $\mathcal{D}_{1}(\neq \emptyset)$ is the solution set of GSNMVIP $(1.5)$ and $\mathcal{L}_{i}: \mathcal{D} \rightarrow \mathcal{D}, i \in\{1,2, \ldots, k\}$ be nonexpansive mapping. Let $\mathcal{K}\left(\mathcal{L}_{i}\right) \neq \emptyset$ be the set of all fixed points of $\mathcal{L}_{i}$. Let $p^{i} \in$ $\mathcal{K}\left(\mathcal{L}_{i}\right)$ and $\left(p^{1}, p^{2}, \ldots p^{k-1}, p^{k}\right) \in \mathcal{D}_{1}$. The fixed point problem (1.6) can be written as

$$
\left\{\begin{array}{l}
p^{1}=\mathcal{L}_{1}\left[p^{1}-g_{1}\left(p^{1}\right)+\mathcal{P}_{\mathcal{D}}\left[f_{1}\left(p^{2}\right)-\lambda_{1} \mathcal{A}_{1}\left(p^{2}, p^{3}, \ldots p^{k}, p^{1}\right)\right]\right] \\
p^{2}=\mathcal{L}_{2}\left[p^{2}-g_{2}\left(p^{2}\right)+\mathcal{P}_{\mathcal{D}}\left[f_{2}\left(p^{3}\right)-\lambda_{2} \mathcal{A}_{2}\left(p^{3}, p^{4}, \ldots p^{1}, p^{2}\right)\right]\right] \\
: \\
: \\
p^{k-1}=\mathcal{L}_{k-1}\left[p^{k-1}-g_{k-1}\left(p^{k-1}\right)+\mathcal{P}_{\mathcal{D}}\left[f_{k-1}\left(p^{k}\right)-\lambda_{k-1} \mathcal{A}_{k-1}\left(p^{k}, p^{1}, \ldots p^{k-2}, p^{k-1}\right)\right]\right] \\
p^{k}=\mathcal{L}_{k}\left[p^{k}-g_{k}\left(p^{k}\right)+\mathcal{P}_{\mathcal{D}}\left[f_{k}\left(p^{1}\right)-\lambda_{k} \mathcal{A}_{k}\left(p^{1}, p^{2}, \ldots p^{k-1}, p^{k}\right)\right]\right] \\
\text { for each } \lambda_{i}>0, i \in\{1,2, \ldots, k\} .
\end{array}\right.
$$

Algorithm 2.1 For any $\left(p_{0}^{1}, p_{0}^{2}, \ldots p_{0}^{k}\right) \in \underbrace{\mathcal{D} \times \mathcal{D} \times \ldots \times \mathcal{D}}_{(\mathrm{k} \text { times })}$, compute the sequences $\left\{p_{n}^{1}\right\},\left\{p_{n}^{2}\right\}, \ldots$ $\left\{p_{n}^{k}\right\}$ by

$$
\left\{\begin{array}{l}
p_{n+1}^{1}=\left(1-\varepsilon_{n}\right) p_{n}^{1}+\varepsilon_{n} \mathcal{L}_{1}\left[p_{n}^{1}-g_{1}\left(p_{n}^{1}\right)+\mathcal{P}_{\mathcal{D}}\left[f_{1}\left(p_{n}^{2}\right)-\lambda_{1} \mathcal{A}_{1}\left(p_{n}^{2}, p_{n}^{3}, \ldots p_{n}^{k}, p_{n}^{1}\right)\right]\right], \\
p_{n+1}^{2}=\mathcal{L}_{2}\left[p_{n+1}^{2}-g_{2}\left(p_{n+1}^{2}\right)+\mathcal{P}_{\mathcal{D}}\left[f_{2}\left(p_{n+1}^{3}\right)-\lambda_{2} \mathcal{A}_{2}\left(p_{n+1}^{3}, p_{n}^{4}, \ldots p_{n}^{1}, p_{n}^{2}\right)\right]\right], \\
: \\
: \\
p_{n+1}^{k-1}=\mathcal{L}_{k-1}\left[p_{n+1}^{k-1}-g_{k-1}\left(p_{n+1}^{k-1}\right)+\mathcal{P}_{\mathcal{D}}\left[f_{k-1}\left(p_{n+1}^{k}\right)-\lambda_{k-1} \mathcal{A}_{k-1}\left(p_{n+1}^{k}, p_{n}^{1}, \ldots p_{n}^{k-2}, p_{n}^{k-1}\right)\right]\right], \\
p_{n+1}^{k}=\mathcal{L}_{k}\left[p_{n+1}^{k}-g_{k}\left(p_{n+1}^{k}\right)+\mathcal{P}_{\mathcal{D}}\left[f_{k}\left(p_{n+1}^{1}\right)-\lambda_{k} \mathcal{A}_{k}\left(p_{n+1}^{1}, p_{n}^{2}, \ldots p_{n}^{k-1}, p_{n}^{k}\right)\right]\right],
\end{array}\right.
$$

where $\lambda_{i}, i \in\{1,2, \ldots, k\}$ and sequence $\varepsilon_{n} \in[0,1]$ for all $n \geq 0$.

If $f_{i}=I, i \in\{1,2, \ldots, k\}$ then Algorithm (2.1) reduces to Algorithm (2.2) as follows:

Algorithm 2.2 For any $\left(p_{0}^{1}, p_{0}^{2}, \ldots p_{0}^{k}\right) \in \underbrace{\mathcal{D} \times \mathcal{D} \times \ldots \times \mathcal{D}}_{(\mathrm{k} \text { times })}$, compute the sequences $\left\{p_{n}^{1}\right\},\left\{p_{n}^{2}\right\}, \ldots$ $\left\{p_{n}^{k}\right\}$ by

$$
\left\{\begin{array}{l}
p_{n+1}^{1}=\left(1-\varepsilon_{n}\right) p_{n}^{1}+\varepsilon_{n} \mathcal{L}_{1}\left[p_{n}^{1}-g_{1}\left(p_{n}^{1}\right)+\mathcal{P}_{\mathcal{D}}\left[p_{n}^{2}-\lambda_{1} \mathcal{A}_{1}\left(p_{n}^{2}, p_{n}^{3}, \ldots p_{n}^{k}, p_{n}^{1}\right)\right]\right], \\
p_{n+1}^{2}=\mathcal{L}_{2}\left[p_{n+1}^{2}-g_{2}\left(p_{n+1}^{2}\right)+\mathcal{P}_{\mathcal{D}}\left[p_{n+1}^{3}-\lambda_{2} \mathcal{A}_{2}\left(p_{n+1}^{3}, p_{n}^{4}, \ldots p_{n}^{1}, p_{n}^{2}\right)\right]\right], \\
: \\
: \\
p_{n+1}^{k-1}=\mathcal{L}_{k-1}\left[p_{n+1}^{k-1}-g_{k-1}\left(p_{n+1}^{k-1}\right)+\mathcal{P}_{\mathcal{D}}\left[p_{n+1}^{k}-\lambda_{k-1} \mathcal{A}_{k-1}\left(p_{n+1}^{k}, p_{n}^{1}, \ldots p_{n}^{k-2}, p_{n}^{k-1}\right)\right]\right], \\
p_{n+1}^{k}=\mathcal{L}_{k}\left[p_{n+1}^{k}-g_{k}\left(p_{n+1}^{k}\right)+\mathcal{P}_{\mathcal{D}}\left[p_{n+1}^{1}-\lambda_{k} \mathcal{A}_{k}\left(p_{n+1}^{1}, p_{n}^{2}, \ldots p_{n}^{k-1}, p_{n}^{k}\right)\right]\right],
\end{array}\right.
$$

where $\lambda_{i}, i \in\{1,2, \ldots, k\}$ and sequence $\varepsilon_{n} \in[0,1]$ for all $n \geq 0$.

If $g_{i}=I, i \in\{1,2, \ldots, k\}$ then Algorithm (2.1) reduces to Algorithm (2.3) as follows:

Algorithm 2.3 For any $\left(p_{0}^{1}, p_{0}^{2}, \ldots p_{0}^{k}\right) \in \underbrace{\mathcal{D} \times \mathcal{D} \times \ldots \times \mathcal{D}}_{(\mathrm{k} \text { times })}$, compute the sequences $\left\{p_{n}^{1}\right\},\left\{p_{n}^{2}\right\}, \ldots$ 
$\left\{p_{n}^{k}\right\}$ by

$$
\left\{\begin{array}{l}
p_{n+1}^{1}=\left(1-\varepsilon_{n}\right) p_{n}^{1}+\varepsilon_{n} \mathcal{L}_{1} \mathcal{P}_{\mathcal{D}}\left[f_{1}\left(p_{n}^{2}\right)-\lambda_{1} \mathcal{A}_{1}\left(p_{n}^{2}, p_{n}^{3}, \ldots p_{n}^{k}, p_{n}^{1}\right)\right], \\
p_{n+1}^{2}=\mathcal{L}_{2} \mathcal{P}_{\mathcal{D}}\left[f_{2}\left(p_{n+1}^{3}\right)-\lambda_{2} \mathcal{A}_{2}\left(p_{n+1}^{3}, p_{n}^{4}, \ldots p_{n}^{1}, p_{n}^{2}\right)\right], \\
: \\
: \\
p_{n+1}^{k-1}=\mathcal{L}_{k-1} \mathcal{P}_{\mathcal{D}}\left[f_{k-1}\left(p_{n+1}^{k}\right)-\lambda_{k-1} \mathcal{A}_{k-1}\left(p_{n+1}^{k}, p_{n}^{1}, \ldots p_{n}^{k-2}, p_{n}^{k-1}\right)\right], \\
p_{n+1}^{k}=\mathcal{L}_{k} \mathcal{P}_{\mathcal{D}}\left[f_{k}\left(p_{n+1}^{1}\right)-\lambda_{k} \mathcal{A}_{k}\left(p_{n+1}^{1}, p_{n+1}^{2}, \ldots p_{n}^{k-1}, p_{n}^{k}\right)\right],
\end{array}\right.
$$

where $\lambda_{i}, i \in\{1,2, \ldots, k\}$ and sequence $\varepsilon_{n} \in[0,1]$ for all $n \geq 0$.

If $f_{i}=g_{i}=I, i \in\{1,2, \ldots, k\}$ then Algorithm (2.1) reduces to Algorithm (2.4) as follows:

Algorithm 2.4 For any $\left(p_{0}^{1}, p_{0}^{2}, \ldots p_{0}^{k}\right) \in \underbrace{\mathcal{D} \times \mathcal{D} \times \ldots \times \mathcal{D}}_{(\mathrm{k} \text { times })}$, compute the sequences $\left\{p_{n}^{1}\right\},\left\{p_{n}^{2}\right\}, \ldots$ $\left\{p_{n}^{k}\right\}$ by

$$
\left\{\begin{array}{l}
p_{n+1}^{1}=\left(1-\varepsilon_{n}\right) p_{n}^{1}+\varepsilon_{n} \mathcal{L}_{1} \mathcal{P}_{\mathcal{D}}\left[p_{n}^{2}-\lambda_{1} \mathcal{A}_{1}\left(p_{n}^{2}, p_{n}^{3}, \ldots p_{n}^{k}, p_{n}^{1}\right)\right] \\
p_{n+1}^{2}=\mathcal{L}_{2} \mathcal{P}_{\mathcal{D}}\left[p_{n+1}^{3}-\lambda_{2} \mathcal{A}_{2}\left(p_{n+1}^{3}, p_{n}^{4}, \ldots p_{n}^{1}, p_{n}^{2}\right)\right] \\
: \\
\vdots \\
p_{n+1}^{k-1}=\mathcal{L}_{k-1} \mathcal{P}_{\mathcal{D}}\left[p_{n+1}^{k}-\lambda_{k-1} \mathcal{A}_{k-1}\left(p_{n+1}^{k}, p_{n}^{1}, \ldots p_{n}^{k-2}, p_{n}^{k-1}\right)\right] \\
p_{n+1}^{k}=\mathcal{L}_{k} \mathcal{P}_{\mathcal{D}}\left[p_{n+1}^{1}-\lambda_{k} \mathcal{A}_{k}\left(p_{n+1}^{1}, p_{n}^{2}, \ldots p_{n}^{k-1}, p_{n}^{k}\right)\right]
\end{array}\right.
$$

where $\lambda_{i}, i \in\{1,2, \ldots, k\}$ and sequence $\varepsilon_{n} \in[0,1]$ for all $n \geq 0$.

If $A_{i}, i \in\{1,2, \ldots, k\}$, is univariate, then Algorithm (2.1) reduces to Algorithm (2.5) as follows:

Algorithm 2.5 For any $\left(p_{0}^{1}, p_{0}^{2}, \ldots p_{0}^{k}\right) \in \underbrace{\mathcal{D} \times \mathcal{D} \times \ldots \times \mathcal{D}}_{k \text { times }}$, compute the sequences $\left\{p_{n}^{1}\right\},\left\{p_{n}^{2}\right\}, \ldots$ $\left\{p_{n}^{k}\right\}$ by

$$
\left\{\begin{array}{l}
p_{n+1}^{1}=\left(1-\varepsilon_{n}\right) p_{n}^{1}+\varepsilon_{n} \mathcal{L}_{1}\left[p_{n}^{1}-g_{1}\left(p_{n}^{1}\right)+\mathcal{P}_{\mathcal{D}}\left[f_{1}\left(p_{n}^{2}\right)-\lambda_{1} \mathcal{A}_{1}\left(p_{n}^{2}\right)\right]\right], \\
p_{n+1}^{2}=\mathcal{L}_{2}\left[p_{n+1}^{2}-g_{2}\left(p_{n+1}^{2}\right)+\mathcal{P}_{\mathcal{D}}\left[f_{2}\left(p_{n+1}^{3}\right)-\lambda_{2} \mathcal{A}_{2}\left(p_{n+1}^{3}\right)\right]\right], \\
: \\
: \\
p_{n+1}^{k-1}=\mathcal{L}_{k-1}\left[p_{n+1}^{k-1}-g_{k-1}\left(p_{n+1}^{k-1}\right)+\mathcal{P}_{\mathcal{D}}\left[f_{k-1}\left(p_{n+1}^{k}\right)-\lambda_{k-1} \mathcal{A}_{k-1}\left(p_{n+1}^{k}\right)\right]\right], \\
p_{n+1}^{k}=\mathcal{L}_{k}\left[p_{n+1}^{k}-g_{k}\left(p_{n+1}^{k}\right)+\mathcal{P}_{\mathcal{D}}\left[f_{k}\left(p_{n+1}^{1}\right)-\lambda_{k} \mathcal{A}_{k}\left(p_{n+1}^{1}\right)\right]\right],
\end{array}\right.
$$

where $\lambda_{i}, i \in\{1,2, \ldots, k\}$ and sequence $\varepsilon_{n} \in[0,1]$ for all $n \geq 0$.

Definition 2.6 A mapping $\mathcal{A}: \underbrace{\mathcal{D} \times \mathcal{D} \times \ldots \mathcal{D}}_{k \text { times }} \rightarrow \mathcal{H}$

(i) is relaxed $(\alpha, \beta)$-cocoercive in the first component if $\exists$ constants $\alpha, \beta>0$ such that

$$
\begin{aligned}
& \left\langle\mathcal{A}_{1}\left(p^{1}, p^{2}, \ldots, p^{k}\right)-\mathcal{A}_{1}\left(p^{1 *}, p^{2 *}, \ldots, p^{k *}\right), p^{1}-p^{1 *}\right\rangle \\
& \quad \geq-\alpha\left\|\mathcal{A}_{1}\left(p^{1}, p^{2}, \ldots, p^{k}\right)-\mathcal{A}_{1}\left(p^{1 *}, p^{2 *}, \ldots, p^{k *}\right)\right\|^{2}+\beta\left\|p^{1}-p^{1 *}\right\|^{2}, \forall p^{1}, p^{1 *} \in \mathcal{D} ;
\end{aligned}
$$


(ii) is $\kappa$-Lipschitz continuous if $\exists$ a constant $\kappa>0$ such that

$$
\left\|\mathcal{A}_{1}\left(p^{1}, p^{2}, \ldots, p^{k}\right)-\mathcal{A}_{1}\left(p^{1 *}, p^{2 *}, \ldots, p^{k *}\right)\right\| \leq \bar{t}\left\|p^{1}-p^{1 *}\right\|, \forall p^{1}, p^{1 *} \in \mathcal{D} .
$$

Lemma 2.7 [18] Let us consider nonnegative sequence $\left\{p_{n}\right\}$, satisfy the following inequality:

$$
p_{n+1} \leq\left(1-\varepsilon_{n}\right) p_{n}+q_{n}, \quad \forall n \geq 0,
$$

with $\varepsilon_{n} \in[0,1], \sum_{n=0}^{\infty} \varepsilon_{n}=\infty$, and $q_{n}=o\left(\varepsilon_{n}\right)$. Then $\lim _{n \rightarrow \infty} p_{n}=0$.

\section{Convergence of k-Steps Iterative Algorithms}

Theorem 3.1 Let $i \in\{1,2, \ldots, k\}$. Assume that $\mathcal{D} \subset \mathcal{H}$ is a closed convex subset in $\mathcal{H}$ and $\mathcal{A}_{i}: \underbrace{\mathcal{D} \times \mathcal{D} \times \ldots \mathcal{D}}_{k \text { times }} \rightarrow \mathcal{H}$ be relaxed $\left(\alpha_{i}, \beta_{i}\right)$-cocoercive and $\kappa_{i}$-Lipschitzian in the first component. Let $g_{i}: \mathcal{D} \rightarrow \mathcal{D}$ be relaxed $\left(r_{i}, s_{i}\right)$-cocoercive and $t_{i}$-Lipschitzian, and $f_{i}$ : $\mathcal{D} \rightarrow \mathcal{D}$ be relaxed $\left(\bar{r}_{i}, \bar{s}_{i}\right)$-cocoercive and $\bar{t}_{i}$-Lipschitzian. Let $\mathcal{L}_{i}: \mathcal{D} \rightarrow \mathcal{D}$ be nonexpansive mapping and $\mathcal{D}_{1} \neq \emptyset, \mathcal{K}\left(\mathcal{L}_{i}\right) \neq \emptyset$. Let $p^{1 *} \in \mathcal{K}\left(\mathcal{L}_{1}\right), p^{2 *} \in \mathcal{K}\left(\mathcal{L}_{2}\right), \ldots, p^{k *} \in \mathcal{K}\left(\mathcal{L}_{k}\right)$ and $\left(p^{1 *}, p^{2 *}, \ldots, p^{k *}\right) \in \mathcal{D}_{1}$. If

$$
\begin{aligned}
& k_{2}<1, k_{3}<1, \ldots, k_{k}<1 \\
& \prod_{i=1}^{k}\left(1-k_{i}\right) \geq \prod_{i=1}^{k}\left(k_{i}^{\prime}+k_{i}^{\prime \prime}\right),
\end{aligned}
$$

where

$$
\begin{aligned}
k_{i} & =\left[1+2 r_{i} t_{i}^{2}-2 s_{i}+t_{i}^{2}\right]^{1 / 2} \\
k_{i}^{\prime} & =\left[1+2 \lambda_{i} \alpha_{i} \kappa_{i}^{2}-2 \lambda_{i} \beta_{i}+\lambda_{i}^{2} \kappa_{i}^{2}\right]^{1 / 2} \\
k_{i}^{\prime \prime} & =\left[1+2 \bar{r}_{i} \bar{t}_{i}^{2}-2 \bar{s}_{i}+\bar{t}_{i}^{2}\right]^{1 / 2}
\end{aligned}
$$

and $\varepsilon_{n} \in[0,1], \sum_{n=0}^{\infty} \varepsilon_{n}=\infty$, then iterative sequences $\left\{p_{n}^{1}\right\},\left\{p_{n}^{2}\right\}, \ldots,\left\{p_{n}^{k}\right\}$ generated by Algorithm (2.1), strongly converges to the solution $\left(p^{1 *}, p^{2 *}, \ldots, p^{k *}\right)$ of GSNMVIP (1.5).

Proof. Let $\left(p^{1 *}, p^{2 *}, \ldots, p^{k *}\right)$ be the solution of GSNMVIP (1.5). By (2.1), we have

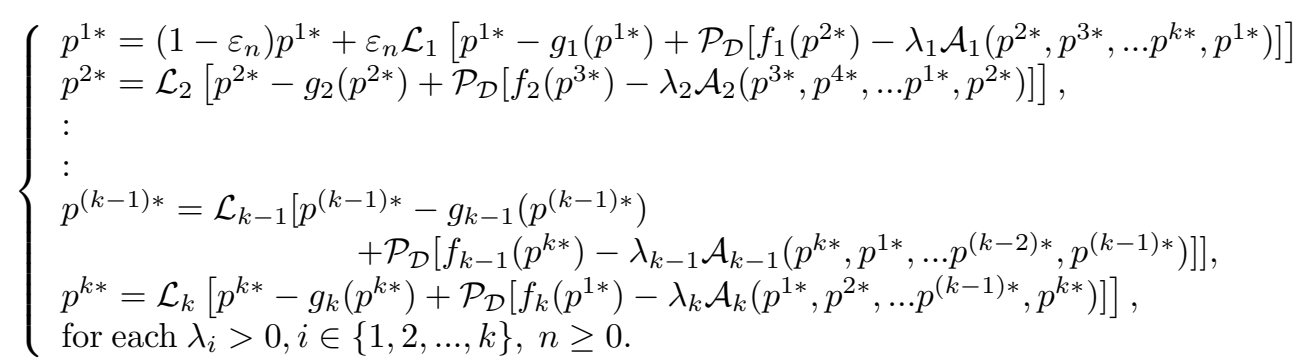


Using Algorithm (2.1) and nonexpansive property of $\mathcal{L}_{i}$ for $i=1$ to evaluate the following

$$
\begin{aligned}
\| p_{n+1}^{1}- & p^{1 *}\|=\|\left(1-\varepsilon_{n}\right) p_{n}^{1}+\varepsilon_{n} \mathcal{L}_{1}\left[p_{n}^{1}-g_{1}\left(p_{n}^{1}\right)+\mathcal{P}_{\mathcal{D}}\left[f_{1}\left(p_{n}^{2}\right)-\lambda_{1} \mathcal{A}_{1}\left(p_{n}^{2}, p_{n}^{3}, \ldots, p_{n}^{k}, p_{n}^{1}\right)\right]\right] \\
& -\left(1-\varepsilon_{n}\right) p^{1 *}-\varepsilon_{n} \mathcal{L}_{1}\left[p^{1 *}-g_{1}\left(p^{1 *}\right)+\mathcal{P}_{\mathcal{D}}\left[f_{1}\left(p^{2 *}\right)-\lambda_{1} \mathcal{A}_{1}\left(p^{2 *}, p^{3 *}, \ldots, p^{k *}, p^{1 *}\right)\right]\right] \| \\
\leq & \left(1-\varepsilon_{n}\right)\left\|p_{n}^{1}-p^{1 *}\right\|+\| \varepsilon_{n}\left[p_{n}^{1}-g_{1}\left(p_{n}^{1}\right)+\mathcal{P}_{\mathcal{D}}\left[f_{1}\left(p_{n}^{2}\right)-\lambda_{1} \mathcal{A}_{1}\left(p_{n}^{2}, p_{n}^{3}, \ldots, p_{n}^{k}, p_{n}^{1}\right)\right]\right] \\
& -\varepsilon_{n}\left[p^{1 *}-g_{1}\left(p^{1 *}\right)+\mathcal{P}_{\mathcal{D}}\left[f_{1}\left(p^{2 *}\right)-\lambda_{1} \mathcal{A}_{1}\left(p^{2 *}, p^{3 *}, \ldots, p^{k *}, p^{1 *}\right)\right]\right] \| \\
\leq & \left(1-\varepsilon_{n}\right)\left\|p_{n}^{1}-p_{n}^{1 *}\right\|+\varepsilon_{n}\left\|p_{n}^{1}-p^{1 *}-\left(g_{1}\left(p_{n}^{1}\right)-g_{1}\left(p^{1 *}\right)\right)\right\| \\
& +\varepsilon_{n}\left\|f_{1}\left(p_{n}^{2}\right)-\lambda \mathcal{A}_{1}\left(p_{n}^{2}, p_{n}^{3}, \ldots, p_{n}^{k}, p_{n}^{1}\right)-\left(f_{1}\left(p^{2 *}\right)-\lambda_{1} \mathcal{A}_{1}\left(p^{2 *}, p^{3 *}, \ldots, p^{k *}, p^{1 *}\right)\right)\right\| \\
\leq & \left(1-\varepsilon_{n}\right)\left\|p_{n}^{1}-p^{1 *}\right\|+\varepsilon_{n}\left\|p_{n}^{1}-p^{1 *}-\left(g_{1}\left(p_{n}^{1}\right)-g_{1}\left(p^{1 *}\right)\right)\right\| \\
& +\varepsilon_{n}\left\|f_{1}\left(p_{n}^{2}\right)-f_{1}\left(p^{2 *}\right)-\lambda_{1}\left(\mathcal{A}_{1}\left(p_{n}^{2}, p_{n}^{3}, \ldots, p_{n}^{k}, p_{n}^{1}\right)-\mathcal{A}_{1}\left(p^{2 *}, p^{3 *}, \ldots, p^{k *}, p^{1 *}\right)\right)\right\| \\
\leq & \left(1-\varepsilon_{n}\right)\left\|p_{n}^{1}-p^{1 *}\right\|+\varepsilon_{n}\left\|p_{n}^{2}-p^{2 *}-\lambda_{1}\left(\mathcal{A}_{1}\left(p_{n}^{2}, p_{n}^{3}, \ldots, p_{n}^{k}, p_{n}^{1}\right)-\mathcal{A}_{1}\left(p^{2 *}, p^{3 *}, \ldots, p^{k *}, p^{1 *}\right)\right)\right\| \\
& +\varepsilon_{n}\left\|p_{n}^{1}-p^{1 *}-\left(g_{1}\left(p_{n}^{1}\right)-g_{1}\left(p^{1 *}\right)\right)\right\|+\varepsilon_{n}\left\|p_{n}^{2}-p^{2 *}-\left(f_{1}\left(p_{n}^{2}\right)-f_{1}\left(p^{2 *}\right)\right)\right\| .
\end{aligned}
$$

Using relaxed $\left(\alpha_{i}, \beta_{i}\right)$-cocoercivity and $\kappa_{i}$-Lipschitz continuity on $\mathcal{A}_{i}$ in the first component for $i=1$, we have

$$
\begin{aligned}
\| p_{n}^{2}- & p^{2 *}-\lambda_{1}\left(\mathcal{A}_{1}\left(p_{n}^{2}, p_{n}^{3}, \ldots, p_{n}^{k}, p_{n}^{1}\right)-\mathcal{A}_{1}\left(p^{2 *}, p_{n}^{3 *}, \ldots, p_{n}^{k *}, p^{1 *}\right)\right) \|^{2} \\
= & \left\|p_{n}^{2}-p^{2 *}\right\|^{2}-2 \lambda_{1}\left\langle\mathcal{A}_{1}\left(p_{n}^{2}, p_{n}^{3}, \ldots, p_{n}^{k}, p_{n}^{1}\right)-\mathcal{A}_{1}\left(p^{2 *}, p^{3 *}, \ldots, p^{k *}, p^{1 *}\right), p_{n}^{2}-p^{2 *}\right\rangle \\
& \quad+\lambda_{1}^{2}\left\|\mathcal{A}_{1}\left(p_{n}^{2}, p_{n}^{3}, \ldots, p_{n}^{k}, p_{n}^{1}\right)-\mathcal{A}_{1}\left(p^{2 *}, p^{3 *}, \ldots, p^{k *}, p^{1 *}\right)\right\|^{2} \\
\leq & \left\|p_{n}^{2}-p^{2 *}\right\|^{2}-2 \lambda_{1}\left[-\alpha_{1}\left\|\mathcal{A}_{1}\left(p_{n}^{2}, p_{n}^{3}, \ldots, p_{n}^{k}, p_{n}^{1}\right)-\mathcal{A}_{1}\left(p^{2 *}, p^{3 *}, \ldots, p^{k *}, p^{1 *}\right)\right\|^{2}\right. \\
& \left.\quad+\beta_{1}\left\|p_{n}^{2}-p^{2 *}\right\|^{2}\right]+\lambda_{1}^{2}\left\|\mathcal{A}_{1}\left(p_{n}^{2}, p_{n}^{3}, \ldots, p_{n}^{k}, p_{n}^{1}\right)-\mathcal{A}_{1}\left(p^{2 *}, p^{3 *}, \ldots, p^{k *}, p^{1 *}\right)\right\|^{2} \\
\leq & \left\|p_{n}^{2}-p^{2 *}\right\|^{2}+2 \lambda_{1} \alpha_{1} \kappa_{1}^{2}\left\|p_{n}^{2}-p^{2 *}\right\|^{2}-2 \lambda_{1} \beta_{1}\left\|p_{n}^{2}-p^{2 *}\right\|^{2}+\lambda_{1}^{2} \kappa_{1}^{2}\left\|p_{n}^{2}-p^{2 *}\right\|^{2} \\
= & {\left[1+2 \lambda_{1} \alpha_{1} \kappa_{1}^{2}-2 \lambda_{1} \beta_{1}+\lambda_{1}^{2} \kappa_{1}^{2}\right]\left\|p_{n}^{2}-p^{2 *}\right\|^{2} } \\
= & k_{1}^{\prime 2}\left\|p_{n}^{2}-p^{2 *}\right\|^{2}, \text { where } k_{1}^{\prime} \text { given by (3.4) for i }=1 .
\end{aligned}
$$

Similarly, by relaxed $\left(r_{i}, s_{i}\right)$-cocoercivity and $t_{i}$-Lipschitz continuity on $g_{i}$ for $i=1$, we have,

$\left\|p_{n}^{1}-p^{1 *}-\left(g_{1}\left(p_{n}^{1}\right)-g_{1}\left(p^{1 *}\right)\right)\right\| \leq k_{1}\left\|p_{n}^{1}-p^{1 *}\right\|$, where $k_{1}$ given by (3.3) for $\mathrm{i}=1 .(3.31)$

Similarly, by relaxed $\left(\bar{r}_{i}, \bar{s}_{i}\right)$-cocoercivity and $\bar{t}_{i}$-Lipschitz continuity on $f_{i}$ for $i=1$, we have,

$\left\|p_{n}^{2}-p^{2 *}-\left(f_{1}\left(p_{n}^{2}\right)-f_{1}\left(p^{2 *}\right)\right)\right\| \leq k_{1}^{\prime \prime}\left\|p_{n}^{2}-p^{2 *}\right\|$, where $k_{1}^{\prime \prime}$ given by (3.5) for $\mathrm{i}=1$ (3. 32)

Using (3.8)-(3.10) in (3.7), we have

$$
\left\|p_{n+1}^{1}-p^{1 *}\right\| \leq\left[1-\left(1-k_{1}\right) \varepsilon_{n}\right]\left\|p_{n}^{1}-p^{1 *}\right\|+\varepsilon_{n}\left(k_{1}^{\prime}+k_{1}^{\prime \prime}\right)\left\|p_{n}^{2}-p^{2 *}\right\| .
$$


By relaxed $\left(\alpha_{i}, \beta_{i}\right)$-cocoercivity and $\kappa_{i}$-Lipschitz continuity on $\mathcal{A}_{i}$ in the first component for $i=2$, we obtain

$$
\begin{aligned}
\| p_{n+1}^{3}- & p^{3 *}-\lambda_{2}\left(\mathcal{A}_{2}\left(p_{n+1}^{3}, p_{n}^{4}, \ldots, p_{n}^{1}, p_{n}^{2}\right)-\mathcal{A}_{2}\left(p^{3 *}, p^{4 *}, \ldots, p^{1 *}, p^{2 *}\right)\right) \|^{2} \\
= & \left\|p_{n+1}^{3}-p^{3 *}\right\|^{2}-2 \lambda_{2}\left\langle\mathcal{A}_{2}\left(p_{n+1}^{3}, p_{n}^{4}, \ldots, p_{n}^{1}, p_{n}^{2}\right)-\mathcal{A}_{2}\left(p^{3 *}, p^{4 *}, \ldots, p^{1 *}, p^{2 *}\right), p_{n+1}^{3}-p^{3 *}\right\rangle \\
& +\lambda_{2}^{2}\left\|\mathcal{A}_{2}\left(p_{n+1}^{3}, p_{n}^{4}, \ldots, p_{n}^{1}, p_{n}^{2}\right)-\mathcal{A}_{2}\left(p^{3 *}, p^{4 *}, \ldots, p^{1 *}, p^{2 *}\right)\right\|^{2} \\
\leq & \left\|p_{n+1}^{3}-p^{3 *}\right\|^{2}-2 \lambda_{2}\left[-\alpha_{2}\left\|\mathcal{A}_{2}\left(p_{n+1}^{3}, p_{n}^{4}, \ldots, p_{n}^{1}, p_{n}^{2}\right)-\mathcal{A}_{2}\left(p^{3 *}, p^{4 *}, \ldots, p^{1 *}, p^{2 *}\right)\right\|^{2}\right. \\
& \left.+\beta_{2}\left\|p_{n+1}^{3}-p^{3 *}\right\|^{2}\right]+\lambda_{2}^{2}\left\|\mathcal{A}_{2}\left(p_{n+1}^{3}, p_{n}^{4}, \ldots, p_{n}^{1}, p_{n}^{2}\right)-\mathcal{A}_{2}\left(p^{3 *}, p^{4 *}, \ldots, p^{1 *}, p^{2 *}\right)\right\|^{2} \\
= & \left.\left\|p_{n+1}^{3}-p^{3 *}\right\|^{2}+2 \lambda_{2} \alpha_{2} \| \mathcal{A}_{2}\left(p_{n+1}^{3}, p_{n}^{4}, \ldots, p_{n}^{1}, p_{n}^{2}\right)-\mathcal{A}_{2}\left(p^{3 *}, p^{4 *}, \ldots, p^{1 *}, p^{2 *}\right)\right) \|^{2} \\
& -2 \lambda_{2} \beta_{2}\left\|p_{n+1}^{3}-p^{3 *}\right\|^{2}+\lambda_{2}^{2}\left\|\mathcal{A}_{2}\left(p_{n+1}^{3}, p_{n}^{4}, \ldots, p_{n}^{1}, p_{n}^{2}\right)-\mathcal{A}_{2}\left(p^{3 *}, p^{4 *}, \ldots, p^{1 *}, p^{2 *}\right)\right\|^{2} \\
\leq & \left\|p_{n+1}^{3}-p^{3 *}\right\|^{2}+2 \lambda_{2} \alpha_{2} \kappa_{2}^{2}\left\|p_{n+1}^{3}-p^{3 *}\right\|^{2}-2 \lambda_{2} \beta_{2}\left\|p_{n+1}^{3}-p^{3 *}\right\|^{2} \\
& +\lambda_{2}^{2} \kappa_{2}^{2}\left\|p_{n+1}^{3}-p^{3 *}\right\|^{2} \\
= & {\left[1+2 \lambda_{2} \alpha_{2} \kappa_{2}^{2}-2 \lambda_{2} \beta_{2}+\lambda_{2}^{2} \kappa_{2}^{2}\right]\left\|p_{n+1}^{3}-p^{3 *}\right\|^{2} } \\
= & k_{2}^{\prime 2}\left\|p_{n+1}^{3}-p^{3 *}\right\|^{2}, \text { where } k_{2}^{\prime} \text { given by (3.4) for i }=2 .
\end{aligned}
$$

Similarly, by relaxed $\left(r_{i}, s_{i}\right)$-cocoercivity and $t_{i}$-Lipschitz continuity on $g_{i}$ for $i=2$, we have,

$\left\|p_{n+1}^{2}-p^{2 *}-\left(g_{2}\left(p_{n+1}^{2}\right)-g_{2}\left(p^{2 *}\right)\right)\right\| \leq k_{2}\left\|p_{n+1}^{2}-p^{2 *}\right\|, k_{2}$ given by (3.3) for $\mathrm{i}=2 .(3.35)$

Similarly, by relaxed $\left(\bar{r}_{i}, \bar{s}_{i}\right)$-cocoercivity and $\bar{t}_{i}$-Lipschitz continuity on $f_{i}$ for $i=2$, we have,

$\left\|p_{n+1}^{3}-p^{3 *}-\left(f_{2}\left(p_{n+1}^{3}\right)-f_{2}\left(p^{3 *}\right)\right)\right\| \leq k_{2}^{\prime \prime}\left\|p_{n+1}^{3}-p^{3 *}\right\|, k_{2}^{\prime \prime}$ given by (3.5) for $\mathrm{i}=2(3.36)$

Using Algorithm (2.1) and nonexpansive property of $\mathcal{L}_{i}$ for $i=2$ to evaluate the following

$$
\begin{aligned}
\| p_{n+1}^{2}- & p^{2 *}\|=\| \mathcal{L}_{2}\left[p_{n+1}^{2}-g_{2}\left(p_{n+1}^{2}\right)+\mathcal{P}_{\mathcal{D}}\left[f_{2}\left(p_{n+1}^{3}\right)-\lambda_{2} \mathcal{A}_{2}\left(p_{n+1}^{3}, p_{n}^{4}, \ldots p_{n}^{1}, p_{n}^{2}\right)\right]\right] \\
& -\mathcal{L}_{2}\left[p^{2 *}-g_{2}\left(p^{2 *}\right)+\mathcal{P}_{\mathcal{D}}\left[f_{2}\left(p^{3 *}\right)-\lambda_{2} \mathcal{A}_{2}\left(p^{3 *}, p^{4 *}, \ldots p^{1 *}, p^{2 *}\right)\right]\right] \| \\
\leq & \|\left[p_{n+1}^{2}-g_{2}\left(p_{n+1}^{2}\right)+\mathcal{P}_{\mathcal{D}}\left[f_{2}\left(p_{n+1}^{3}\right)-\lambda_{2} \mathcal{A}_{2}\left(p_{n+1}^{3}, p_{n}^{4}, \ldots p_{n}^{1}, p_{n}^{2}\right)\right]\right] \\
& -\left[p^{2 *}-g_{2}\left(p^{2 *}\right)+\mathcal{P}_{\mathcal{D}}\left[f_{2}\left(p^{3 *}\right)-\lambda_{2} \mathcal{A}_{2}\left(p^{3 *}, p^{4 *}, \ldots, p^{1 *}, p^{2 *}\right)\right] \|\right. \\
\leq & \left\|p_{n+1}^{2}-p^{2 *}-\left(g_{2}\left(p_{n+1}^{2}\right)-g_{2}\left(p^{2 *}\right)\right)\right\|+\| \mathcal{P}_{\mathcal{D}}\left[f_{2}\left(p_{n+1}^{3}\right)-\lambda_{2} \mathcal{A}_{2}\left(p_{n+1}^{3}, p_{n}^{4}, \ldots p_{n}^{1}, p_{n}^{2}\right)\right] \\
& -\mathcal{P}_{\mathcal{D}}\left[f_{2}\left(p^{3 *}\right)-\lambda_{2} \mathcal{A}_{2}\left(p^{3 *}, p^{4 *}, \ldots, p^{1 *}, p^{2 *}\right)\right] \| \\
\leq & \left\|p_{n+1}^{2}-p^{2 *}-\left(g_{2}\left(p_{n+1}^{2}\right)-g_{2}\left(p^{2 *}\right)\right)\right\|+\|\left[f_{2}\left(p_{n+1}^{3}\right)-\lambda_{2} \mathcal{A}_{2}\left(p_{n+1}^{3}, p_{n}^{4}, \ldots p_{n}^{1}, p_{n}^{2}\right)\right] \\
& -\left[f_{2}\left(p^{3 *}\right)-\lambda_{2} \mathcal{A}_{2}\left(p^{3 *}, p^{4 *}, \ldots, p^{1 *}, p^{2 *}\right)\right] \| \\
\leq & \left\|p_{n+1}^{3}-p^{3 *}-\lambda_{2}\left(\mathcal{A}_{2}\left(p_{n+1}^{3}, p_{n}^{4}, \ldots, p_{n}^{1}, p_{n}^{2}\right)-\mathcal{A}_{2}\left(p^{3 *}, p^{4 *}, \ldots, p^{1 *}, p^{2 *}\right)\right)\right\| \\
& +\left\|p_{n+1}^{3}-p^{3 *}-\left(f_{2}\left(p_{n+1}^{3}\right)-f_{2}\left(p^{3 *}\right)\right)\right\|+\left\|p_{n+1}^{2}-p^{2 *}-\left(g_{2}\left(p_{n+1}^{2}\right)-g_{2}\left(p^{2 *}\right)\right)\right\|
\end{aligned}
$$


Using (3.12)-(3.14) in (3.15), we have

$$
\begin{aligned}
& \left\|p_{n+1}^{2}-p^{2 *}\right\| \leq\left\{k_{2}^{\prime}+k_{2}^{\prime \prime}\right\}\left\|p_{n+1}^{3}-p^{3 *}\right\|+k_{2}\left\|p_{n+1}^{2}-p^{2 *}\right\| \\
& \left\|p_{n+1}^{2}-p^{2 *}\right\| \leq \frac{\left(k_{2}^{\prime}+k_{2}^{\prime \prime}\right)}{1-k_{2}}\left\|p_{n+1}^{3}-p^{3 *}\right\|, \text { where } k_{2}<1 \text { by }(3.1),
\end{aligned}
$$

that is

$$
\left\|p_{n}^{2}-p^{2 *}\right\| \leq \frac{\left(k_{2}^{\prime}+k_{2}^{\prime \prime}\right)}{1-k_{2}}\left\|p_{n}^{3}-p^{3 *}\right\|, \text { where } k_{2}<1 \text { by }(3.1) .
$$

Through this similar process, we can evaluate

$$
\begin{gathered}
\left\|p_{n}^{3}-p^{3 *}\right\| \leq \frac{\left(k_{3}^{\prime}+k_{3}^{\prime \prime}\right)}{1-k_{3}}\left\|p_{n}^{4}-p^{4 *}\right\|, \text { where } k_{3}<1 \text { by }(3.1) \\
: \\
: \\
\left\|p_{n}^{k-1}-p^{(k-1) *}\right\| \leq \frac{\left(k_{k-1}^{\prime}+k_{k-1}^{\prime \prime}\right)}{1-k_{k-1}}\left\|p_{n}^{k}-p^{k *}\right\|, \text { where } k_{k-1}<1 \text { by }(3.1) \\
\left\|p_{n}^{k}-p^{k *}\right\| \leq \frac{\left(k_{k}^{\prime}+k_{k}^{\prime \prime}\right)}{1-k_{k}}\left\|p_{n}^{1}-p^{1 *}\right\|, \text { where } k_{k}<1 \text { by }(3.1)
\end{gathered}
$$

Using (3.11), (3.16)-(3.19), we get

$$
\begin{gathered}
\left\|p_{n+1}^{1}-p^{1 *}\right\| \leq\left(1-\varepsilon_{n}\left(1-k_{1}-\left(k_{1}^{\prime}+k_{1}^{\prime \prime}\right) \frac{\left(k_{2}^{\prime}+k_{2}^{\prime \prime}\right)}{1-k_{2}} \frac{\left(k_{3}^{\prime}+k_{3}^{\prime \prime}\right)}{1-k_{3}} \ldots \frac{\left(k_{k-1}^{\prime}+k_{k-1}^{\prime \prime}\right)}{1-k_{k-1}} \frac{\left(k_{k}^{\prime}+k_{k}^{\prime \prime}\right)}{1-k_{k}}\right)\right) \\
\times\left\|p_{n}^{1}-p^{1 *}\right\| . \\
\left\|p_{n+1}^{1}-p^{1 *}\right\| \leq\left(1-\varepsilon_{n}\left(1-k_{1}-\left(k_{1}^{\prime}+k_{1}^{\prime \prime}\right) \prod_{i=2}^{k} \frac{\left(k_{i}^{\prime}+k_{i}^{\prime \prime}\right)}{1-k_{i}}\right)\right)\left\|p_{n}^{1}-p^{1 *}\right\| .(3.42)
\end{gathered}
$$

Since

$$
\left(1-k_{1}-\left(k_{1}^{\prime}+k_{1}^{\prime \prime}\right) \prod_{i=2}^{k} \frac{\left(k_{i}^{\prime}+k_{i}^{\prime \prime}\right)}{1-k_{i}}\right) \in[0,1]
$$

and $\sum_{n=0}^{\infty} \varepsilon_{n}=\infty$, from Lemma 2.7, we have $\lim _{n \rightarrow \infty}\left\|p_{n}^{1}-p^{1 *}\right\|=0$. This completes the proof.

Corollary 3.2 Let $i \in\{1,2, \ldots, k\}$. Assume that $\mathcal{D} \subset \mathcal{H}$ is a closed convex subset in $\mathcal{H}$ and $\mathcal{A}_{i}: \underbrace{\mathcal{D} \times \mathcal{D} \times \ldots \mathcal{D}}_{k \text { times }} \rightarrow \mathcal{H}$ be relaxed $\left(\alpha_{i}, \beta_{i}\right)$-cocoercive and $\kappa_{i}$-Lipschitzian in the first component, and $g_{i}: \mathcal{D} \rightarrow \mathcal{D}$ be relaxed $\left(r_{i}, s_{i}\right)$-cocoercive and $t_{i}$-Lipschitzian. Let 
$\mathcal{L}_{i}: \mathcal{D} \rightarrow \mathcal{D}$ be nonexpansive mapping and $\mathcal{D}_{2} \neq \emptyset, \mathcal{K}\left(\mathcal{L}_{i}\right) \neq \emptyset$. Let $p^{1 *} \in \mathcal{K}\left(\mathcal{L}_{1}\right), p^{2 *} \in$ $\mathcal{K}\left(\mathcal{L}_{2}\right), \ldots, p^{k *} \in \mathcal{K}\left(\mathcal{L}_{k}\right)$ and $\left(p^{1 *}, p^{2 *}, \ldots, p^{k *}\right) \in \mathcal{D}_{2}$. If

$$
\begin{aligned}
& k_{2}<1, k_{3}<1, \ldots, k_{k}<1 \\
& \prod_{i=1}^{k}\left(1-k_{i}\right) \geq \prod_{i=1}^{k} k_{i}^{\prime}
\end{aligned}
$$

where

$$
\begin{aligned}
k_{i} & =\left[1+2 r_{i} t_{i}^{2}-2 s_{i}+t_{i}^{2}\right]^{1 / 2} \\
k_{i}^{\prime} & =\left[1+2 \lambda_{i} \alpha_{i} \kappa_{i}^{2}-2 \lambda_{i} \beta_{i}+\lambda_{i}^{2} \kappa_{i}^{2}\right]^{1 / 2}
\end{aligned}
$$

and $\varepsilon_{n} \in[0,1], \sum_{n=0}^{\infty} \varepsilon_{n}=\infty$, then iterative sequences $\left\{p_{n}^{1}\right\},\left\{p_{n}^{2}\right\}, \ldots,\left\{p_{n}^{k}\right\}$ generated by Algorithm (2.2), strongly converges to the solution $\left(p^{1 *}, p^{2 *}, \ldots, p^{k *}\right)$ of GSNMVIP (1.7).

Corollary 3.3 Let $i \in\{1,2, \ldots, k\}$. Assume that $\mathcal{D} \subset \mathcal{H}$ is a closed convex subset in $\mathcal{H}$ and $\mathcal{A}_{i}: \underbrace{\mathcal{D} \times \mathcal{D} \times \ldots \mathcal{D}}_{k \text { times }} \rightarrow \mathcal{H}$ be relaxed $\left(\alpha_{i}, \beta_{i}\right)$-cocoercive and $\kappa_{i}$-Lipschitzian in the first component, and $f_{i}: \mathcal{D} \rightarrow \mathcal{D}$ be relaxed $\left(\bar{r}_{i}, \bar{s}_{i}\right)$-cocoercive and $\bar{t}_{i}$-Lipschitzian. Let $\mathcal{L}_{i}: \mathcal{D} \rightarrow \mathcal{D}$ be nonexpansive mapping and $\mathcal{D}_{3} \neq \emptyset, \mathcal{K}\left(\mathcal{L}_{i}\right) \neq \emptyset$. Let $p^{1 *} \in \mathcal{K}\left(\mathcal{L}_{1}\right), p^{2 *} \in$ $\mathcal{K}\left(\mathcal{L}_{2}\right), \ldots, p^{k *} \in \mathcal{K}\left(\mathcal{L}_{k}\right)$ and $\left(p^{1 *}, p^{2 *}, \ldots, p^{k *}\right) \in \mathcal{D}_{3}$. If

$$
\prod_{i=1}^{k}\left(k_{i}^{\prime}+k_{i}^{\prime \prime}\right) \leq 1
$$

where

$$
\begin{aligned}
& k_{i}^{\prime}=\left[1+2 \lambda_{i} \alpha_{i} \kappa_{i}^{2}-2 \lambda_{i} \beta_{i}+\lambda_{i}^{2} \kappa_{i}^{2}\right]^{1 / 2} \\
& k_{i}^{\prime \prime}=\left[1+2 \bar{r}_{i} \bar{t}_{i}^{2}-2 \bar{s}_{i}+\bar{t}_{i}^{2}\right]^{1 / 2}
\end{aligned}
$$

and $\varepsilon_{n} \in[0,1], \sum_{n=0}^{\infty} \varepsilon_{n}=\infty$, then iterative sequences $\left\{p_{n}^{1}\right\},\left\{p_{n}^{2}\right\}, \ldots,\left\{p_{n}^{k}\right\}$ generated by Algorithm (2.3), strongly converges to the solution $\left(p^{1 *}, p^{2 *}, \ldots, p^{k *}\right)$ of GSNMVIP (1.9).

Corollary 3.4 Let $i \in\{1,2, \ldots, k\}$. Assume that $\mathcal{D} \subset \mathcal{H}$ is a closed convex subset in $\mathcal{H}$ and $\mathcal{A}_{i}: \underbrace{\mathcal{D} \times \mathcal{D} \times \ldots \mathcal{D}}_{k \text { times }} \rightarrow \mathcal{H}$ be relaxed $\left(\alpha_{i}, \beta_{i}\right)$-cocoercive and $\kappa_{i}$-Lipschitzian in the first component. Let $\mathcal{L}_{i}: \mathcal{D} \rightarrow \mathcal{D}$ be nonexpansive mapping and $\mathcal{D}_{4} \neq \emptyset, \mathcal{K}\left(\mathcal{L}_{i}\right) \neq \emptyset$. Let $p^{1 *} \in \mathcal{K}\left(\mathcal{L}_{1}\right), p^{2 *} \in \mathcal{K}\left(\mathcal{L}_{2}\right), \ldots, p^{k *} \in \mathcal{K}\left(\mathcal{L}_{k}\right)$ and $\left(p^{1 *}, p^{2 *}, \ldots, p^{k *}\right) \in \mathcal{D}_{4}$. If

$$
\prod_{i=1}^{k} k_{i}^{\prime} \leq 1
$$

where

$$
k_{i}^{\prime}=\left[1+2 \lambda_{i} \alpha_{i} \kappa_{i}^{2}-2 \lambda_{i} \beta_{i}+\lambda_{i}^{2} \kappa_{i}^{2}\right]^{1 / 2}
$$


and $\varepsilon_{n} \in[0,1], \sum_{n=0}^{\infty} \varepsilon_{n}=\infty$, then iterative sequences $\left\{p_{n}^{1}\right\},\left\{p_{n}^{2}\right\}, \ldots,\left\{p_{n}^{k}\right\}$ generated by Algorithm (2.4), strongly converges to the solution $\left(p^{1 *}, p^{2 *}, \ldots, p^{k *}\right)$ of GSNMVIP (1.11).

Corollary 3.5 Let $i \in\{1,2, \ldots, k\}$. Assume that $\mathcal{D} \subset \mathcal{H}$ is a closed convex subset in $\mathcal{H}$ and univariate mappings $\mathcal{A}_{i}: \mathcal{D} \rightarrow \mathcal{H}$ be relaxed $\left(\alpha_{i}, \beta_{i}\right)$-cocoercive and $\kappa_{i}$-Lipschitzian. Let $g_{i}: \mathcal{D} \rightarrow \mathcal{D}$ be relaxed $\left(r_{i}, s_{i}\right)$-cocoercive and $t_{i}$-Lipschitz, and $f_{i}: \mathcal{D} \rightarrow \mathcal{D}$ be relaxed $\left(\bar{r}_{i}, \bar{s}_{i}\right)$-cocoercive and $\bar{t}_{i}$-Lipschitz. Let $\mathcal{L}_{i}: \mathcal{D} \rightarrow \mathcal{D}$ be nonexpansive mapping and $\mathcal{D}_{5} \neq \emptyset, \mathcal{K}\left(\mathcal{L}_{i}\right) \neq \emptyset$. Let $p^{1 *} \in \mathcal{K}\left(\mathcal{L}_{1}\right), p^{2 *} \in \mathcal{K}\left(\mathcal{L}_{2}\right), \ldots, p^{k *} \in \mathcal{K}\left(\mathcal{L}_{k}\right)$ and $\left(p^{1 *}, p^{2 *}, \ldots, p^{k *}\right) \in \mathcal{D}_{5}$. If

$$
\begin{aligned}
& k_{2}<1, k_{3}<1, \ldots, k_{k}<1 \\
& \prod_{i=1}^{k}\left(1-k_{i}\right) \geq \prod_{i=1}^{k}\left(k_{i}^{\prime}+k_{i}^{\prime \prime}\right),
\end{aligned}
$$

where

$$
\begin{aligned}
& k_{i}=\left[1+2 r_{i} t_{i}^{2}-2 s_{i}+t_{i}^{2}\right]^{1 / 2} \\
& k_{i}^{\prime}=\left[1+2 \lambda_{i} \alpha_{i} \kappa_{i}^{2}-2 \lambda_{i} \beta_{i}+\lambda_{i}^{2} \kappa_{i}^{2}\right]^{1 / 2} \\
& k_{i}^{\prime \prime}=\left[1+2 \bar{r}_{i} \bar{t}_{i}^{2}-2 \bar{s}_{i}+\bar{t}_{i}^{2}\right]^{1 / 2}
\end{aligned}
$$

and $\varepsilon_{n} \in[0,1], \sum_{n=0}^{\infty} \varepsilon_{n}=\infty$, then iterative sequences $\left\{p_{n}^{1}\right\},\left\{p_{n}^{2}\right\}, \ldots,\left\{p_{n}^{k}\right\}$ generated by Algorithm (2.5), strongly converges to the solution $\left(p^{1 *}, p^{2 *}, \ldots, p^{k *}\right)$ of GSNMVIP (1.13).

Remark 3.6 If $k=1$ and $g_{i}=\mathcal{L}_{i}=I_{i}$, then Theorem 3.1 reduces to Noor's result discussed in [9]. If $k=1,2, A_{i}=A$ and $f_{i}=g_{i}=\mathcal{L}_{i}=I$, then Theorem 3.1 reduces to Verma's result discussed in [16]. In addition, $A_{i}$ is univariate mapping then Theorem 3.1 reduces to Verma's result discussed in in $[15,17]$. If $k=1,2$, and $f_{i}=\mathcal{L}_{i}=I$, then Theorem 3.1 reduces to Noor's result discussed in [8]. If $k=1,2, f_{i}=g_{i}$ and $\mathcal{L}_{i}=I$, then Theorem 3.1 reduces to Hao's result discussed in [5]. If $k=1,2,3$ and $\mathcal{L}_{i}=I$, then Theorem 3.1 reduces to Zhang's result discussed in [19].

\section{Conclusion}

In this paper, we suggest and analyze $k$-steps iterative method for finding the approximate solution of generalized systems of nonlinear mixed variational inequalities including $3 \mathrm{k}$ distinct nonlinear relaxed cocoercive operators in real Hilbert space, which can be viewed as a refinement and improvement of some existing results on solving many systems of variational inequality problems (e.g. [5, 8, 9, 15, 16, 17, 19]). Some of them existing results can be viewed as special cases of Algorithm 2.1. Therefore, the new algorithm is expected to be widely applicable. 


\section{Acknowledgment}

The author would like to thank the editor and anonymous referees for their outstanding suggestions and nice comments that improve the presentation of this manuscript.

\section{References}

[1] J.P. Aubin, A. Cellina Differential inclusions, Springer-Verlag, Berlin, (1984).

[2] A. Bnouhachem, A modified projection method for a common solution of a system of variational inequalities, a split equilibrium problem and a hierarchical fixed-point problem, Fixed Point Theory Appl. 22, (2014) 1-25.

[3] A. Bnouhachem, M.A. Noor, M. Khalfaoui and S. Zhaohan, A resolvent algorithm of generalized system for relaxed cocoercive mixed variational inequalities, Appl. Comput. Math., 12, No. 2 (2013) 125-132.

[4] A. Bnouhachem, M.A. Noor, M. Khalfaoui and H. Benazza, Generalized system of variational inequalities in Banach spaces, Appl. Math. Inf. Sci. 8, No. 3 (2014) 985991.

[5] Y. Hao X. Qin and S.M. Kang, Systems of relaxed cocoercive generalized variational inequalities via nonexpansive mappings, Math. Commun. 16, (2011) 179-190.

[6] K.S. Kim, System of extended general variational inequalities for relaxed cocoercive mappings in Hilbert space, Mathematics, 6, No. 10 (2018) 1-12.

[7] D. Kinderlehrer, G. Stampacchia, An introduction to variational inequalities and their applications, Academic Press, New York, USA, (1980).

[8] M.A. Noor, K. Inayat Noor, Projection algorithms for solving a system of general variational inequalities, Nonlinear Anal. 70, (2009) 2700-2706.

[9] M.A. Noor, Differentiable nonconvex functions and general variational inequalities, Appl. Math. Comput. 199, (2008) 623-630.

[10] M. A. Noor, Extended general variational inequalities, Appl. Math. Lett. 22, (2009) 182-186.

[11] M. A. Noor, On a class of general variational inequalities, J. Adv. Math. Studies 1, (2009) 75-85.

[12] M.A. Noor, K.I. Noor and A. Bnouhachem, On a unified implicit method for variational inequalities, Journal of Comput. and Appl. Math., 249, (2013) 6973.

[13] M.A. Noor, K.I. Noor, A.G. Khan, R. Latif, Some new algorithms for solving a system of general variational inequalities. Appl. Math. Inf. Sci. 10, (2016) 18. 
[14] G. Stampacchia, Formes bilineaires coercivities sur les ensembles convexes, C. R. Acad. Sci. Paris, 258, (1964) 4413-4416.

[15] R.U. Verma, Projection methods, algorithms, and a new system of nonlinear variational inequalities, Comput. Math. Appl. 41, (2001) 1025-1031.

[16] R.U. Verma, Generalized system for relaxed cocoercive variational inequalities and projection methods, J. Optim. Theory Appl. 121, (2004) 203-210.

[17] R.U. Verma, General convergence analysis for two-step projection methods and applications to variational problems, Appl. Math. Lett. 18, (2005) 1286-1292.

[18] X.L. Weng, Fixed point iteration for local strictly pseudocontractive mappings, Proc. Amer. Math. Soc. 113, (1991) 727-731.

[19] M. Zhang, Iterative algorithms for a system of generalized variational inequalities in Hilbert spaces, Fixed Point Theory Appl. 232, (2012) 1-13. 